\title{
Electron Ptychography: From 2D to 3D Reconstructions
}

Si Gao ${ }^{1}$, Fucai Zhang ${ }^{2,3,4}$, A. I. Kirkland ${ }^{2,5,6}$, Xiaoqing Pan $^{7}$ and Peng Wang ${ }^{1}$

${ }^{1 .}$ National Laboratory of Solid State Microstructures, College of Engineering and Applied Sciences and Collaborative Innovation Center of Advanced Microstructures, Nanjing University, Nanjing, People's Republic of China.

2. Research Complex at Harwell, Harwell Oxford Campus, Didcot, UK.

${ }^{3 .}$ London Centre for Nanotechnology, London, UK.

4. Department of Electrical and Electronic Engineering, Southern University of Science and Technology, Shenzhen, China.

5. Department of Materials, University of Oxford, Parks Road, Oxford, UK.

6. Electron Physical Sciences Imaging Centre, Diamond Lightsource, Diamond House, Oxfordshire, Didcot, UK

${ }^{7 .}$ Department of Physics and Astronomy, University of California, Irvine, CA, USA

Transmission electron microscopy (TEM) is a powerful tool for material science characterisation due to its high spatial resolution. Coherent diffraction imaging (CDI) [1] is a "lensless" method that forms an image of an object by solving the phase problem from a single diffraction pattern with an iterative phasing algorithm. This approach can, in principle, overcome the current image resolution limiting factors and ultimately achieve wavelength-limited resolution. However, conventional CDI experiments require isolated samples and a priori knowledge about the sample shape or extent. Maiden and Rodenburg suggested an extended ptychographical iterative engine (ePIE), which does not need this prior information and overcomes many of the other issues of CDI, such as convergence stagnation and limited field of view. Recently, two-dimensional (2D) ptychographical reconstructions at atomic lateral resolution [2] have been achieved using ePIE [3] on a $\mathrm{CeO}_{2}$ nanoparticle. However, when the sample becomes thicker, the multiplicative assumption of electron-sample interaction becomes invalid causing the ptychographical reconstruction to breakdown. To overcome this limitation, Maiden et al. incorporated the well-known multiple slice solution to multiple scattering into the ePIE algorithm (3PIE) [4]. In this approach the sample is split into axial sections, to produce 3D optically sectioned images of the sample. This 3D multiple-section reconstruction of ptychography has been successfully demonstrated with both light [4] and X-ray [5] optics recently. However, this method is still a challenge for use with the electrons.

In this work, firstly we will demonstrate the possibility of achieving 2D phase reconstructions of nanoparticles $\left(\mathrm{LaB}_{6}\right)$ at sub-Angström resolution using the ePIE algorithm, as shown Fig. 1. Secondly we will show a successful 3D reconstruction of nanostructured materials using 3PIE [4] based on the multislice approach for the first time. The 3D experiment shown in Fig. 2 a) was carried out at $60 \mathrm{kV}$ using a (S)TEM Titan ${ }^{3}$ instrument fitted with a field emission source. The probe-forming aperture with a semi-angle of $22 \mathrm{mrad}$ was used and 400 diffraction images were acquired. Using the 3PIE reconstruction algorithm we were able to obtain the complex object function of the sample at multislices along the sample thickness direction as shown in Fig. 2 c). The reconstructed 3D information of the sample is consistent with its TEM image as shown in Fig. 2 b) [6]. 


\section{References:}

[1] R Gerchberg and WO Saxton, Optik 35 (1972), p. 237.

[2] AJ D’Alfonso et al, Physical Review B 89 (2014), p. 064101.

[3] AM Maiden and JM Rodenburg, Ultramicroscopy 109 (2009), p. 1256.

[4] AM Maiden, MJ Humphry and JM Rodenburg, Journal of the Optical Society of America a-Optics Image Science and Vision 29 (2012), p. 1606.

[5] A Suzuki, S Furutaku and K Shimomura, et al, Physical Review Letters 112 (2014), p. 053903.

[6] The authors acknowledge funding from the National Natural Science Foundation of China

(11474147), the National Basic Research Program of China, (Grant No. 2015CB654900) and the International Science \& Technology Cooperation Program of China (2014DFE00200).
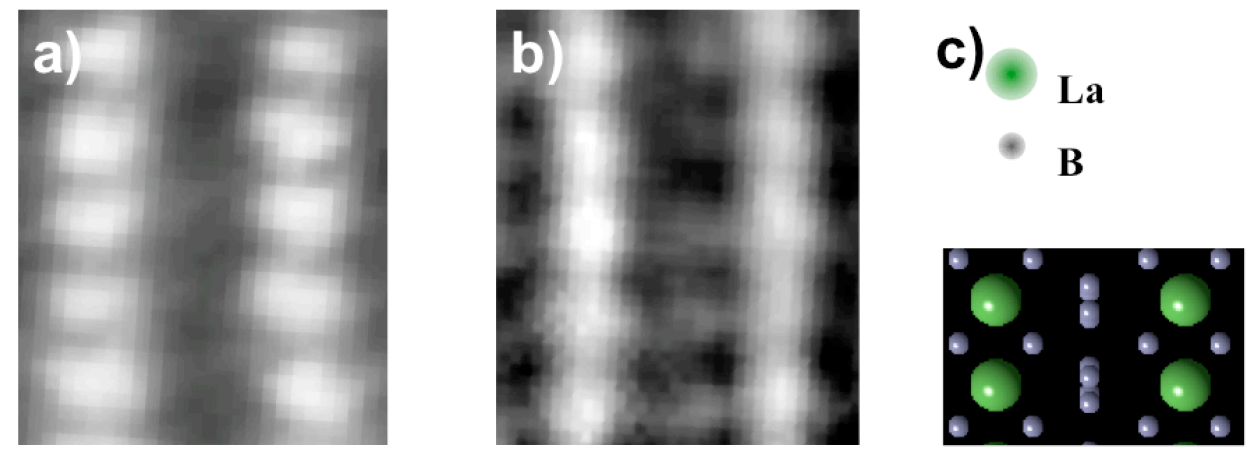

Figure 1. a) HAADF image of a $\mathrm{LaB}_{6}$ crystal; b) reconstructed phase of a $\mathrm{LaB}_{6}$ crystal; c) Projected atomic models of $\mathrm{LaB}_{6}$ along $<210>$.
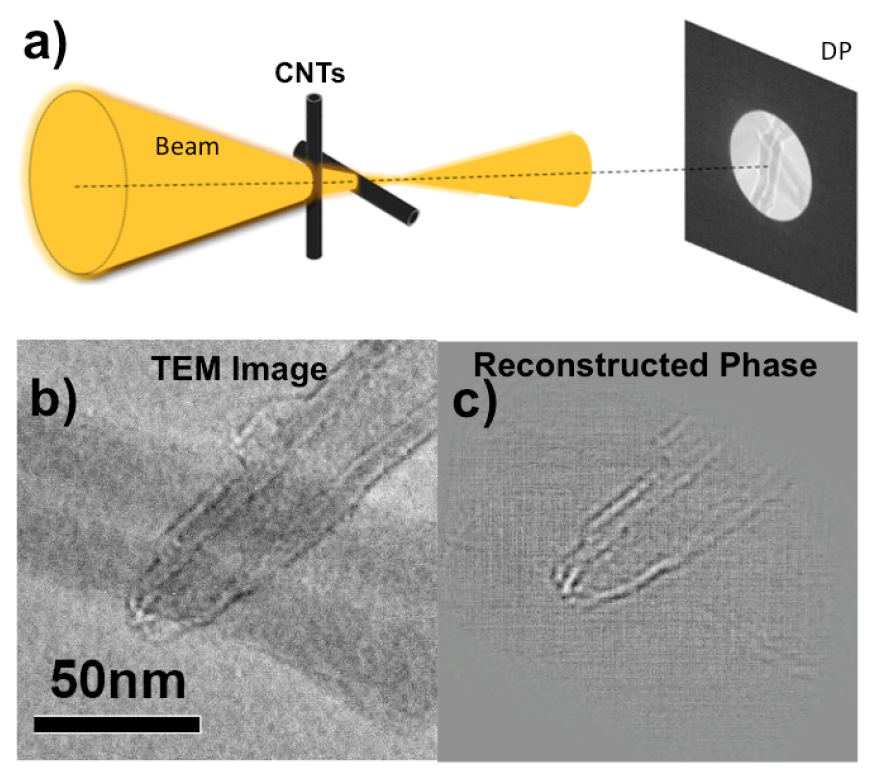

Figure 2. a) A schematic of the electron optical geometry used for 3D ptychography. The electron beam propagates through two CNT bundles at different sample heights along the optical axis and far field diffraction patterns are recorded. b) Conventional TEM image of CNTs; c) Reconstructed phase of one of the slices showing the upper CNT. 